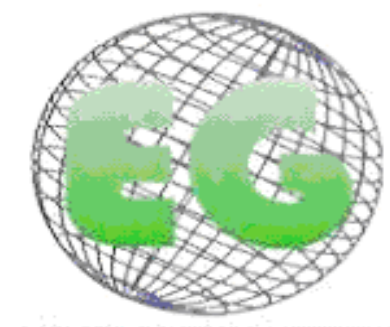

ISSN 1695-6141 $N^{\circ} 26$

\title{
Envejeciendo con AIDS 0 el AIDS en el envejecimiento: epidemiológico en un hospital de la Universidad de Rio de Janeiro
}

perfil Envelhecendo com Aids ou a Aids no envelhecimento: perfil epidemiológico em um Hospital Universitario do Rio de Janeiro

\section{*Lombardo Pereira, G., " Costa Aguiar, BG.}

\author{
*Profa Adjunta. E-mail gilombardo@hotmail.com **Profa Associada. Departamento de Enfermagem Médico \\ Cirúrgica, Escola de Enfermagem Alfredo Pinto, Universidade Federal do Estado do Rio de Janeiro. Brasil.
}

Palabras clave: mujeres; anciano; HIV; perfil epidemiológico.

Palavras chave: mulheres; idoso; HIV; perfil epidemiológico.

Keywords: women; elderly; HIV; epidemiological profile

\section{RESUMEN}

El estudio tiene como objetivo identificar el perfil epidemiológico del VIH / SIDA en la población de mujeres de 60 años y más de un Hospital Universitario y describir los elementos que caracterizan a la infección en esta población, teniendo en cuenta las cuestiones de género. Esta investigación documental pretende presentar las características de la infección de estas mujeres. Se seleccionó una muestra empírica de la población atendida en el hospital. Esta muestra fue representativa para reflejar su comportamiento.

Los resultados permitieron conocer el perfil epidemiológico de la clientela como analizaron los registros de 106 individuos mayores de 60 años, 57 varones y 49 mujeres. De todos los registros se excluyeron aquellos que no contenían datos relativos a la escolaridad, estado civil, fecha de diagnóstico (confirmación de laboratorio) y la forma de contaminación. Se analizaron 34 registros.

Se concluyó que el perfil de las mujeres mayores de 60 años en el Hospital Universitario confirma los datos actuales en el país, destacando de este grupo la proporción de mujeres solteras y viudas, el $29 \%$ y $35 \%$ respectivamente, que se infectaron por relaciones heterosexuales, caracterizando la incidencia en las mujeres que estaban solas.

\section{RESUMO}

O estudo objetiva identificar o perfil epidemiológico de HIV/AIDS na população de mulheres 60 anos e mais de um Hospital Universitario e descrever as evidências que caracterizam a infecção nesta população considerando as questões de gênero. Trata-se de pesquisa documental, onde se buscou apresentar as características que envolveram a infecção destas mulheres. Seleciou-se uma amostra empírica da população atendida no hospital. Esta amostra foi representativa de modo a refletir seu comportamento. 
Os resultados propiciaram conhecer o perfil epidemiológico da clientela visto que foram analisados 106 prontuarios de indivíduos com 60 anos e mais, sendo 57 masculinos e 49 femininos. Do total de prontuarios femininos excluíram-se aqueles que não continham dados referentes à escolaridade; estado civil; data do diagnóstico (confirmação laboratorial) e a forma de contaminação. Analisou-se, então, 34 prontuários.

Concluiu-se que o perfil das mulheres de 60 anos e mais atendidas no Hospital Universitario confirma os dados atuais no país, sendo destacado deste grupo a proporcionalidade de mulheres solteiras e viúvas, $29 \%$ e $35 \%$ respectivamente, que se contaminaram em relações heterossexuais, caracterizando a incidencia em mulheres que encontravam-se sós.

\section{ABSTRACT}

The study aims to identify the epidemiological profile of HIV / AIDS in the population of women of 60 years and more at a University Hospital and to describe the elements characterizing the infection in this population taking into account considering gender issues. This documentary research seeks to, to present the characteristics involved in the infection of these women. An empirical sample was selected from the population treated at the hospital. The sample was representative, in order to reflect behavior.

The results revealed the epidemiological profile of the clientele from the reds of 106 individuals, aged 60 and over, 57 male and 49 female. Excluded were women who did not contain data related to education, marital status, date of diagnosis (laboratory confirmation) and the form of contamination. 34 records were then analysed, concluding that the profile of women aged 60 and older at the Hospital Universitario confirms the current data in the country, and highlighting within this group the proportion of single women and widows, $29 \%$ and $35 \%$ respectively, who are infected by heterosexual relations, and characterizing the incidence in women who were alone.

\section{INTRODUCCIÓN}

La población mundial de personas con 60 años o más crece gradualmente. En Brasil creció "en casi cinco décadas más del doble, dado que en el año 1959 eran aproximadamente 204 millones de ancianos en el mundo y en 1998, 579 millones. Teniendo una previsión de 1.900 milllones para el año 2050" 1 .

El envejecimiento poblacional es una conquista oriunda de la evolución científica, tecnológica y social. Lo que viene desafiando todos los sectores sociales y la sociedad de modo general para atender a estos individuos con dignidad y responsabilidad favoreciendo su mayor autonomía ${ }^{2}$.

En Brasil, la "legislación de protección social ha avanzado mucho" ${ }^{3}$, teniendo como marco principal la elaboración del Estatuto del Anciano. Más recientemente el Ministerio de Salud divulgó el Manual del Anciano ${ }^{4}$, asegurando ser la longevidad "un triunfo" del mundo actual, cuyas proyecciones para el año 2050, en Brasil y en el mundo es que "existirán más ancianos que niños menores de 15 años, fenómeno nunca antes observado".

En este contexto del envejecimiento de la población mundial se encuentra un problema, el AIDS, que por sus características viene creciendo en este grupo poblacional. EI AIDS es aún un grave problema de Salud Colectiva, por referirse a los procesos de salud dolencia, sufrimiento-muerte en la sociedad ${ }^{5}$. Es una enfermedad caracterizada por una disfunción grave del sistema inmunológico del individuo infectado por el HIV ${ }^{6}$. La llegada de la terapia antirretroviral asociada con la adherencia al tratamiento proporcionó una mejora en la calidad de vida y consecuentemente mayor longevidad de la persona infectada. 
Conforme el Ministerio de Salud ${ }^{7}$ el AIDS es una enfermedad emergente, que representa uno de los mayores problemas de salud de la actualidad, debido a su carácter pandémico y a su gravedad.

Este problema surgió en la década de 80 afectando letalmente a individuos jóvenes en fase de producción económica y de reproducción de la especie humana. Todavía la introducción de los antirretrovirales de alto valor de acción, la participación efectiva de los gobiernos en el combate a la epidemia y las actividades educativas de carácter preventivo, en el fin de la década, favorecieron el control más eficaz de la replicación viral en el organismo del individuo y de las enfermedades oportunistas contribuyendo a la longevidad de estos infectados con mejor calidad de vida.

Frente a estos hechos se tiene la Política Nacional de Salud de la Persona Anciana ${ }^{8}$ (PNSPI) que propone una condición de independencia de la capacidad funcional abarcando no solamente el ámbito de la "salud física y mental", sino también "las condiciones socioeconómicas y de capacidad de auto cuidado".

Siendo así, ¿cómo articular sistemáticamente las acciones preconizadas en el Pacto por la Vida con las directrices establecidas en la PNSPI a los portadores de HIV/AIDS de modo a alcanzar la tan ansiada independencia? ¿Y qué independencia es esta que garantiza a la población anciana las acciones recomendadas en el Pacto por la Vida?

Estos cuestionamientos reclaman investigar el perfil epidemiológico de la epidemia entre la población femenina anciana. Además de requerir una presentación de estrategias que permitan relacionar las propuestas de la PNSPI, principalmente en lo que se refiere a "promoción del envejecimiento activo y saludable y atención integral, integrada a la salud de la persona anciana" 8 .

Así el objeto del estudio consistirá en la participación de la enfermera en la identificación del perfil epidemiológico de la epidemia del AIDS entre mujeres con 60 años y más, a fin de buscar estrategias de orientación y control.

El aumento de la población de 60 años y más es comprobado a través de los datos estadísticos del DATASUS ${ }^{9}$, donde el índice de envejecimiento, o sea, el número de personas ancianas para cada 100 individuos jóvenes por año en Brasil, que era en 2000, 28.9, en 2005 se cuentan significativamente 33.9, debido a la consecuente elevación de la "Esperanza de Vida" por mejores condiciones de vida asegurada por el Sistema Único de Salud (SUS) ${ }^{10}$ y consolidada a través de la primera ley dirigida a la salud del anciano - LEY 8.842, corroborada actualmente con la Política Nacional de Salud de la Persona Anciana.

Además, la esperanza de vida a los 60 años de edad salta, en 2000 de 19,66 a 20,82, en 2004, asegurando lo expuesto en el Art. 9o del Capítulo I (Del Derecho a la Vida), Título II (De los Derechos Fundamentales, del Estatuto del Anciano ${ }^{8}$ que cita:"Es obligación del Estado, garantizar a la persona anciana la protección a la vida y a la salud, mediante realización de políticas sociales públicas que permitan un envejecimiento saludable y en condiciones de dignidad".

Otro hecho importante a destacar son los datos estadísticos de mortalidad apuntando que el sexo masculino muere en mayor cantidad que el femenino, evidenciando que el envejecimiento también alimenta la cuestión de género. Con todo, este predominio del contingente femenino más expresivo cuanto más anciano sea el segmento, ocurre en zonas 
urbanas. "En las rurales, predominan los hombres, lo que puede resultar en aislamiento y abandono de esas personas". ${ }^{8}$

A partir de estos datos el objeto del estudio son los factores que contribuyen al aumento de casos de HIV/AIDS entre mujeres de 60 años y más, al haber un mayor número de infectados por el HIV/AIDS en el sexo femenino que masculino.

De esta forma el estudio busca identificar la ocurrencia de la infección por HIV/AIDS a partir de los sesenta años de edad, en la considerada tercera edad, cuando estas mujeres, al terminar una relación buscan o encuentran otro compañero y en este momento se contaminan.

Fueron formulados los siguientes objetivos para clarear los hechos destacados:

1) Identificar el perfil epidemiológico de HIV/AIDS en la población de mujeres ancianas en una institución hospitalaria de enseñanza, a través de datos de los prontuarios.

2) Describir las evidencias que caracterizan la infección en esta población específica considerando las cuestiones de género.

3) Constatar, por medio de los prontuarios, el período de la infección en estas mujeres ancianas, o sea, a partir de los 60 años.

Este estudio se torna relevante en el sentido de evidenciar los factores que determinan la longevidad como un posible condicionante del aumento en el número de personas infectadas por el HIV. Caracterizando, también, una mayor proporción de la infección entre las mujeres de 60 años y más de edad estimulando identificar el perfil en esta población con destaque a las cuestiones de género.

Para la asistencia de enfermería reside en percibir el comportamiento de la persona anciana ante la infección por el HIV. Así como realizar una evaluación de la calidad de su asistencia.

De esta manera, el estudio podrá contribuir para el medio académico fortaleciendo el panorama actual, en el área de la salud de la mujer, en cuanto a la infección por el HIV. De esta forma posibilitará la reflexión de los profesionales en formación acerca del saber y de la importancia de una asistencia de calidad.

En relación a la investigación, el estudio sugiere nuevos objetos para ser desarrollados, considerando la magnitud del proceso de la infección por el HIV y el envejecimiento.

\section{METODOLOGÍA}

El estudio es de tipo exploratorio con abordaje cualitativo. "Los estudios exploratorios permiten al investigador aumentar su experiencia en torno a determinado problema". ${ }^{11}$

Confrontando la citación y el tipo de estudio este se consolida con lo expuesto a través de sus objetivos, principalmente, en la identificación del perfil epidemiológico del HIV/AIDS.

Pautado en esta afirmación, el estudio buscó describir la realidad presentada por los trazos característicos que envolvieron la infección por el HIV/AIDS en las mujeres con sesenta años, acompañadas en la unidad hospitalaria de enseñanza seleccionada. 
El escenario de pesquisa fue el ambulatorio de inmunología de un Hospital Universitario, localizado en la Zona Norte del Municipio del Rio de Janeiro, donde presta asistencia integrada con las directrices y propuestas de la Política Nacional de Salud a Persona Anciana y del Programa Nacional de Aids.

Los sujetos fueron mujeres de 60 años y más portadoras del HIV/AIDS atendidas en el referido ambulatorio, a fin de identificar el perfil cuanto al proceso en que ocurrió la infección por el HIV/AIDS relacionando al proceso de envejecimiento.

Los datos fueron colectados en prontuarios médicos, conforme con los objetivos del estudio, visto que "ellos facilitarán idea de las características a ser observadas o medidas" ${ }^{12}$. i

Para un mejor esclarecimiento es importante enfatizar que la representatividad es el reflejo del su comportamiento en la población, allende tener una buena aproximación de los parámetros poblacionales correspondientes; caso contrario, se corre el riesgo de ver el proceso de inferencia comprometido, al generar estimativas proporcionadas por los parámetros. $^{12}$

Así, siendo la representatividad para el estudio en cuestión dependieron de la dimensión poblacional encontrada a través de los prontuarios de modo a corresponder el reflejo y los parámetros de la población de mujeres ancianas portadoras do HIV/AIDS atendidas en el Ambulatorio.

La colecta de los datos evidenció el proceso de direccionamiento actual del Aids en la población. Esto puede también subsidiar acciones del cuidar de enfermería diferenciada y sistematizada, abrir la puerta a otras investigaciones que proporcionarán resultados de proporciones tan interesantes e importantes del cuidar preventivo.

Brasil registró, en números absolutos, 192.709 óbitos por AIDS, en el período de 1980 a $200{ }^{13}$. Aún en esta contextualización histórica a lo largo del tiempo, la razón entre los sexos viene disminuyendo de forma progresiva. En 1985, había 15 casos de la enfermedad en hombres por 1 en mujer. Hoy, la relación es de 1,5 a 1. En el grupo de edad de 13 a 19 años, hubo una inversión en la razón de sexo, a partir de 1998.

Se observa en la literatura que la mayor parte de los casos se concentran en el grupo de edad de 25 a 49 años. Sin embargo, en los últimos años, se ha verificado aumento del porcentual de casos en la población mayor de 50 años, en ambos sexos. Por lo tanto, este estudio busca identificar el perfil epidemiológico de la población atendida en el sentido de evidenciar la relación de este grupo destacado (mujeres ancianas) con los casos del grupo de edad de mayor concentración.

La colecta de datos fue obtenida a través de los registros sistemáticos de los prontuarios de las mujeres de 60 años y más portadoras de HIV/AIDS atendidas en el ambulatorio del Hospital Universitario del Rio de Janeiro, en el período de marzo a julio de 2009. Se utilizó un guión previamente elaborado, con el compromiso de mantener toda condición de privacidad.

A fin de atender a la mejora de la calidad de vida se utilizó el instrumento abreviado de evaluación de la calidad de vida - WHOQOL-Bref (versión brasileña) para las mujeres con 60 años y más portadoras de HIV/Aids. Este instrumento abreviado sometido a criterios de selección que ayudan en la evaluación de la calidad de vida de la persona anciana complementó la pesquisa. 
De acuerdo con el Grupo de Calidad de Vida de la Organización Mundial de la Salud ${ }^{14}$ calidad de vida es "la percepción del individuo de su posición en la vida, en el contexto de la cultura y sistema de valores en los cuales vive y en relación a s sus objetivos, expectativas, padrones y preocupaciones".

Según FLECK ${ }^{14}$, inicialmente "fue desarrollado un instrumento compuesto con cien (100) cuestiones - WHOQOL -100". Entretanto, para atender y envolver la participación de varios países se crearon versiones que considerasen las diferencias culturales de cada país y no se despreciase la característica principal, como es el caso de la versión brasileña WHOQOL-Bref. Allende esta preocupación existe la necesidad de instrumentos cortos que demanden "poco tiempo para su cumplimiento", así como no omiten la metodología y características de satisfacción para medir la calidad de vida.

Ante lo expuesto se torna evidente presentar los dominios y facetas del WHOQOL-bref para interacción y entendimiento del proceso de evaluación a ser utilizado en este estudio. Son contemplados cuatro dominios: físico, psicológico, relaciones social y medio ambiente.

El dominio físico se detiene en las evidencias y condiciones de dolor e incomodidad, energía y fatiga; sueño y reposo; movilidad; dependencia de medicación o de tratamiento; y, capacidad de trabajo. El dominio psicológico relaciona los sentimientos positivos; el pensar, el aprender, la memoria y concentración; la autoestima; la imagen corporal y aspecto; los sentimientos negativos; y, la espiritualidad / religión / creencias personales. El tercer dominio, relaciones sociales, envuelve las relaciones personales, o sea, soporte (apoyo) social y actividad sexual. Y, por fin, el medio ambiente, que perpetúa a través de la seguridad física y protección; ambiente en lar, recursos financieros; cuidados de salud y sociales, disponibilidad y calidad; oportunidades de adquirir nuevas informaciones y habilidades; participación en y oportunidades de recreación y ocio; ambiente físico, tales como: polución, ruido, tránsito y clima; y, transporte.

Las facetas para puntuación son seis y cada una posee cuatro ítems que consiste en un factor de orden más elevado en los análisis factoriales confirmatorios que son: la menor probabilidad de dolencia, la alta capacidad funcional física y mental y el compromiso social activo con la vida. En el conjunto de perfil de seis escores de facetas puede haber un único escore total basado en la suma de todos los 24 ítems del módulo.

Se concluye que un "envejecimiento bien sucedido puede ser entendido a partir de sus tres componentes: (a) menor probabilidad de enfermedad; (b) alta capacidad funcional física y mental; $y$ (c) compromiso social activo con la vida". 8

Mediante lo expuesto, el instrumento utilizado para la colecta de datos se pautó en algunos datos del WHOQOL-bref y de la "Ficha de Notificación de Aids en Adulto" elaborado por el Ministerio de la Salud.

La apropiación del WHOQOL-bref fue para asociar la afirmativa de que la prevalencia del HIV en la población general se mantiene estabilizada, estando Brasil en la posición de países con "epidemia concentrada "entre la población de 15 a 49 años, siendo 0,42\% entre las mujeres". 9

A través del instrumento fue posible evaluar el perfil de las mujeres de 60 años y más atendidas en el Ambulatorio del Hospital Universitario y verificar que el mismo coincide con el cuadro epidemiológico de Brasil. Los datos facilitarán las características del perfil epidemiológico de esta clientela específica. 


\section{RESULTADO}

Los datos fueron obtenidos del archivo de prontuarios del ambulatorio de infectología donde fueron consultados 106 (ciento y seis) prontuarios de individuos con 60 años y más, siendo 57 masculinos y 49 femeninos. Del total de prontuarios femeninos se excluyeron aquellos que no contuvieron datos referentes a la escolaridad; estado civil; y fecha del diagnóstico, o sea, fecha de confirmación laboratorial. Seleccionandose, entonces, 34 prontuarios para el análisis.

De los 34 prontuarios analizados se destaca que las mujeres ancianas atendidas en la unidad se encuentran, en su mayoría, en el grupo de edad comprendido entre 60 y 69 años, correspondiendo a $82 \%$, conforme gráfico 1 .

Gráfico 1: Porcentual por faja etária de mujeres ancianas con hiv atendidas en el Ambulatorio de Infectología del Hospital Universitario

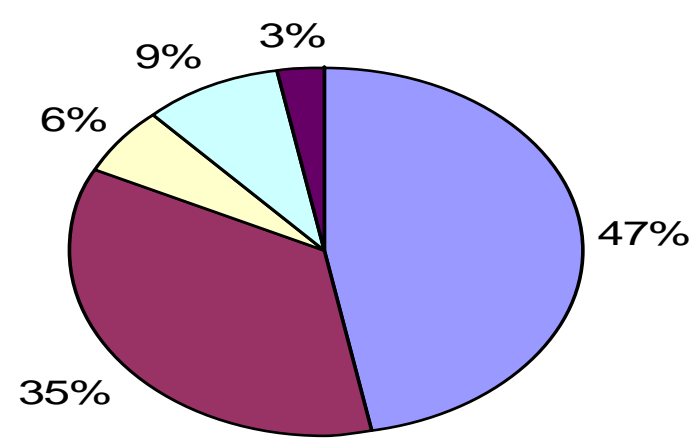

$\square 60-64 \square 65-69 \square 70-74 \quad \square 75-79 \square 80-99$

FUENTE: Prontuarios del Ambulatorio de Inmunología del Hospital Universitario, mar.jun., 2009.

Se constató, también, que la contaminación de estas mujeres ocurrió en relación heterosexual en $85 \%$ de los casos. Lo que refuerza el incentivo al uso del preservativo, independiente del grupo de edad. 
Gráfico 2: Forma de contaminación de las mujeres de 60 años y más con hiv atendidas en el Hospital Universitario.

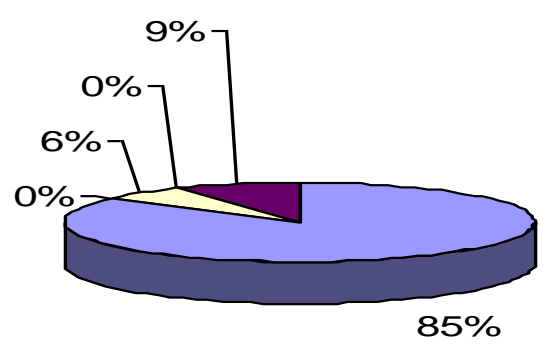

$$
\begin{aligned}
& \square \text { Heterossexual } \\
& \square \text { Homossexual } \\
& \square \text { Hemotransfusão } \\
& \square \text { Uso Drogas } \\
& \text { Injetáveis } \\
& \square \text { Ignorado }
\end{aligned}
$$

FUENTE: Prontuarios del Ambulatorio de Infectología del Hospital Universitario, mar./jun., 2009.

El grado de instrucción y la ocupación, en el grupo de edad de esta pesquisa, consolidan los indicadores que apuntan la pobreza, o la cuestión del empobrecimiento de las personas como factores condicionantes de la infección por el HIV. El indicador escolaridad es utilizado como uno de los indicadores más importantes para medir el nivel socioeconómico asociado a la salud de la población" ${ }^{15}$. Así, el estudio, también, constata que la evolución de la epidemia en Brasil presenta inclinación a la feminización y empobrecimiento.

Gráfico 3: Grado de instrucción de las mujeres de 60 años y más con hiv atendidas en el Hospital Universitario.

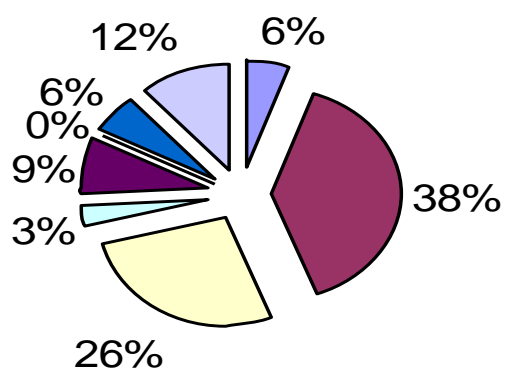

$\square$ Analfabeto
$\square$ Fundamental Completo
$\square$ Médio Completo
$\square$ Superior Completo

$\square$ Fundamental Incompleto

$\square$ Médio Incompleto

$\square$ Superior Incompleto

$\square$ Ignorado

FUENTE: Prontuarios del Ambulatorio de Infectología del Hospital Universitario, mar./jun., 2009. 
El estudio evidenció que el perfil de las mujeres de 60 años y más atendidas en el Hospital Universitario confirma los datos del direccionamiento actualmente en el país, siendo destacado de este grupo, del estudio, la proporcionalidad de mujeres solteras y viudas, $29 \%$ y $35 \%$ respectivamente, que se contaminaron en relaciones heterosexuales, caracterizando la incidencia mayor en mujeres que se encontraban, en algún momento, solas.

Allende esta evidencia, el estudio comprobó que la mayoría (59\%) de las mujeres se infectaron en el grupo de edad entre 50 y 59 años de edad, o sea, antes de insertarse en la edad determinada por la Política Nacional de Atención a Persona Anciana lo que genera preocupación en conocer el proceso por el cual pasa la infección del HIV/AIDS.

Gráfico 4 - Edad de las mujeres correspondiente a fecha del diagnóstico para HIV/AIDS

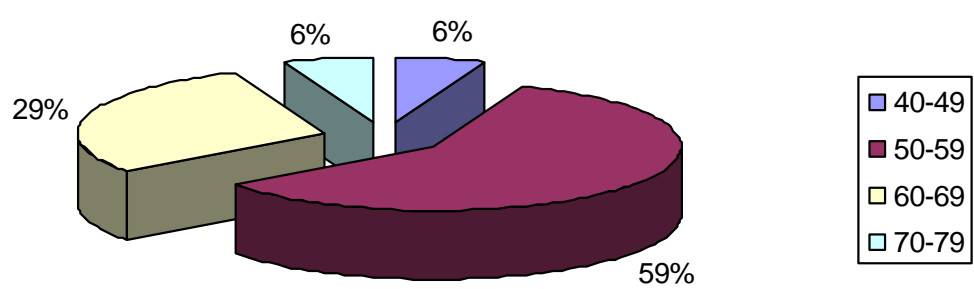

FUENTE: Prontuarios del Ambulatorio de Infectología del Hospital Universitario, mar./jun., 2009.

Como el envejecimiento es un proceso que ocurre naturalmente, es preciso que la sociedad esté atenta a la mejora de la calidad de vida de la población anciana para que esta ocurra de forma "activa, libre de cualquier tipo de dependencia funcional, lo que exige promoción de la salud en todas las edades" 8.

El hecho de que la epidemia, hoy, presenta una estabilización en su prevalencia está asociado a cambio de comportamiento, prácticas y actitudes, de la población brasileña frente a las cuestiones relacionadas a la transmisión del HIV ${ }^{9}$ por conocimiento de las formas de transmisión.

Un hecho importante para esta estabilización fue el uso del preservativo, principalmente, entre los jóvenes que pasaron a utilizarlo en la primera relación sexual o con compañero eventual lo que sugiere un direccionamiento, entre la población de 60 años y más, de actividad de orientación y estímulo a la práctica del uso del preservativo.

\section{CONCLUSIÓN}

En este estudio al analizarse el perfil de las mujeres de 60 años y más atendidas en el Hospital Universitario se constata que los datos confirman los que están siendo divulgados actualmente en el país y, es menester destacar de este grupo que hay una proporcionalidad entre mujeres solteras y viudas, $29 \%$ y $35 \%$ respectivamente, que se contaminaron en relaciones heterosexuales, caracterizando que la infección ocurre con mayor incidencia en 
mujeres que se encontraban, en algún momento, solas. Además de evidenciar que la contaminación aconteció en el $59 \%$ de los casos antes que las mujeres entraran en la tercera edad reforzando las cuestiones de género. .

El estudio trajo, por consiguiente reflexiones para otras pesquisas con esta clientela, tales como: ¿las mujeres de 60 años y más estarían utilizando el preservativo en una relación eventual? ¿El nuevo compañero acepta usar el preservativo? ¿Cómo estimular a estas mujeres a obligar el uso del preservativo por el compañero y con esto obtener menor probabilidad de enfermedad, alta capacidad funcional física y mental y comprometimiento social activo con la vida, principalmente ante la infección por el HIV cuando se encuentran solas e inician una nueva relación?

\section{Agradecimlentos}

Nuestro agradecimento por su participación:

Márcia Lima Costa - Enfermera del Hospital Universitario Gaffrée e Guinle da UNIRIO Cristiane de Sousa Silva - Enfermera formada por la Escola de Enfermagem Alfredo Pinto, UNIRIO. Entonces era Estudiante

Raquel Medina Ribeiro - Enfermera formada por la Escola de Enfermagem Alfredo Pinto, UNIRIO. Entonces era Estudiante Natália Gonçalves Mateus - Enfermera formada por la Escola de Enfermagem Alfredo Pinto, UNIRIO. Entonces era Estudiante.

\section{REFERENCIAS BIBLIOGRÁFICAS}

1. Instituto Brasileiro de Geografia e Estatística. Perfil dos idosos responsáveis pelos domicílios no Brasil. População. Projeção da População do Brasil por sexo e idade para o período 1980-2050. Consulta através do site: http://www.ibge.gov.br. Em 02/11/2007.

2. Brasil. Ministerio da Saúde. Secretaria Executiva. Departamento de Apoio à Descentralização. Coordenação geral de Apoio à Gestão Descentralizada. Diretrizes operacionais dos Pactos pela Vida, Defesa do SUS e de Gestão. 2ª ed. Brasília. 2006.

3. Telles JL. Perspectivas e Desafios para o Planejamento das Ações do Pacto pela Vida/Saúde da População Idosa 2006/2007: Contribuição para o Debate. DAPE/SAS/MS. Brasília, nov. 2006.

4. Brasil. Secretaria de Atenção a Saúde. Departamento de Atenção Básica. Envelhecimento e saúde da pessoa idosa. Caderno de Atenção Básica - no․ 9. Ministério da Saúde. Secretaria de Atenção a Saúde. Departamento de Atenção Básica. Brasília, 2006

5. Paim JS e Almeida Filho NA Crise da Saúde Pública e a Utopia da Saúde Coletiva. Salvador, Bahia: Casa da Qualidade Editora, 2000.

6. Brasil. Ministério da Saúde: Fundação Nacional de Saúde Doenças infecciosas e parasitárias: aspectos clínicos de vigilância epidemiológica e de controle. guia de bolso. Elaborado por Gerson de Oliveira Pena [et al] 2ª ed. Brasília: Ministério da Saúde: Fundação Nacional de Saúde, 2000.

7. Brasil. Fundação Nacional de Saúde. Guia de vigilância epidemiológica / Fundação Nacional de Saúde. 5ํㅡㄹ ed. Brasília: FUNASA, 2002.

8. Brasil. Ministério da Saúde. Política Nacional de Saúde da Pessoa Idosa. Portaria GM no․ 2528 de 19/10/2006.

9. Brasil. Programa Nacional DST e Aids. Resposta - Experiências do Programa Brasileiro de Aids. PASCOM, Ana Roberta Pati [et al]. Epidemia de aids no Brasil. Tendências e 
comportamento relacionado ao HIV/aids. [acesso em 2005 Ago 09] Disponível em: http://www.aids.gov.br..

10. Brasil. Sistema Único de Saúde. Lei 8.080. [acesso em 2007 Ago 20]. Disponível em: http://www6.senado.gov.br/legislacao/ListaPublicacoes. action?id=134238.

11. Triviños A N S. Introdução à pesquisa em ciências sociais qualitativa em educação. São Paulo: Atlas, 1987. pág. $109-114$.

12. Medronho RA. Epidemiologia. São Paul: Editora Atheneu, 2004. pág. 283 - 334.

13. Brasil. Ministério da Saúde. Portal de Serviços do Governo. Boletim Epidemiológico 2008. [acesso em 2009 Fev 09]. Disponível em: www. Aids.gov.br/data/Pages/LUMIS13F4BF21PTBRIE.htm.

14. Fleck PA, Louzada S, Xavier M, Chechemovich E, Vieira G, Santos L. Aplicação da versão em português do instrumento abreviado de avaliação da qualidade de vida "WHOQOL-Bref". Rev S Pub. 2000; 34(2): 178-183. [acesso em 2009 Set 27]. Disponível em: www.fsp.usp.br/rsp

15. Fonseca MG. In: Padoin SMM. Aids: o que ainda há para ser dito? Santa Maria: ed. UFSM, 2000. 